J. Clin. Chem. Clin. Biochem.

Vol. 17, 1979, pp. 1-7

\title{
Peroxidase aus Meerrettich: Kinetische Studien sowie Optimierung der Aktivitätsbestimmung mit den Substraten $\mathrm{H}_{2} \mathrm{O}_{2}$ und 2,2'-Azino-di-(3-ethyl-benzthiazolinsulfonsäure-(6)) (ABTS)
}

\author{
Von H. Gallati \\ Diagnostische Forschungsabteilung der F. Hoffmann-La Roche \& Co. AG Basel (Schweiz)
}

(Eingegangen am 19. Juni / 5. August 1978)

Zusammenfassung: Die kinetischen Studien der Peroxidase aus Meerrettich konzentrieren sich vor allem auf die Abhängigkeit der Enzymaktivität von der $\mathrm{H}_{2} \mathrm{O}_{2}$ - und der ABTS-Konzentration bei verschiedenen pH-Werten. Dabei werden die Michaelis-Konstanten für die beiden Substrate bestimmt und in Korrelation zum pH gesetzt. Die Resultate zeigen, daß die Affinität von ABTS zur Peroxidase mit steigendem pH-Wert abnimmt, die pH-Optima mit höheren ABTS-Konzentrationen gegen den Neutralpunkt verschoben werden, und die maximale Reaktionsgeschwindigkeit beim pH 4,2 mit einer ABTS- Konzentration von $2 \mathrm{mmol} / \mathrm{l}$ erreicht wird.

Aus den kinetischen Daten des $\mathrm{H}_{2} \mathrm{O}_{2}$ wird deutlich, daß das dissozierte $\mathrm{O}_{2} \mathrm{H}^{-}$als eigentlicher Wasserstoff-Akzeptor bei der Peroxidasereaktion angesehen werden muß. Bei der Komplexbildung mit der Peroxidase konkurrieren ABTS und $\mathrm{H}_{2} \mathrm{O}_{2}$, wodurch die Michaelis-Konstanten der beiden Substrate gegenseitig beeinflußt werden.

Zur Optimierung der Aktivitätsbestimmung der Peroxidase werden die Einflüsse der Puffersysteme, des pH-Wertes, der Konzentrationen von ABTS und $\mathrm{H}_{2} \mathrm{O}_{2}$ sowie der Reaktionsdauer auf die Aktivität dèr Peroxidase untersucht und auf Grund dieser Ergebnisse ein Testverfahren vorgeschlagen, das erlaubt, die Peroxidase bis zu einer Konzentration von $10 \mathrm{ng} / 1$ zu bestimmen.

Horseradish peroxidase: $A$ study of the kinetics and the determination of optimal reaction conditions, using hydrogen peroxide and 2,2'-azinobis 3-ethylbenzthiazoline-6-sulfonic acid (ABTS) as substrates

Summary: The reaction of the two substrates hydrogen peroxide and ABTS with horseradish peroxidase was studied kinetically. Enzyme activity was determined as a function of substrate concentration and $\mathrm{pH}$. Michaelis constants were determined for the two substrates at various $\mathrm{pH}$ values. It was found that the affinity of the enzyme for ABTS decreases with increasing $\mathrm{pH}$, and that with higher $\mathrm{ABTS}$ concentrations the $\mathrm{pH}$ optima of the reaction are shifted towards neutrality. Maximal rate is reached at $\mathrm{pH} 4.2$ with an ABTS concentration of $2 \mathrm{mmol} / \mathrm{l}$.

For hydrogen peroxide the data show that the dissociated $\mathrm{O}_{2} \mathrm{H}^{-}$is the proper substrate, its affinity for the enzyme being independent of $\mathrm{pH}$. The two substrates show competitive binding to the peroxidase, and each therefore influences the binding constant of the other.

A procedure is proposed which allows the determination of peroxidase down to a concentration of $10 \mathrm{ng} / \mathrm{l}$ or $2.5 \times 10^{-13} \mathrm{~mol} / 1$.

\section{Einleitung}

Die Peroxidase aus Meerrettich (Donor: hydrogen peroxide oxidoreductase, EC 1.11.1.7) wird häufig bei den enzym-immunologischen Bestimmungen als Markierungsund Indikatorenzym verwendet. Daher ist eine einfache Methode zur quañtitātiven Erfassung kleinster Peroxidasemengen von entscheidendèr Bedeutung.

In der vorliegenden Arbeit werden die katalytischen Eigenschaften der Peroxidäse aus Meerrettich untersucht, um Einblick in den Wirkungsmechanismus dieses
Enzyms z̃u erhalten. Diese Untersuchungen betreffen vor allem die Abhängigkeit der Peroxidaseaktivität von der Konzentration der beiden Substrate $\mathrm{H}_{2} \mathrm{O}_{2}$ als Wasserstoff-Akzeptor und des Redoxindikators 2,2'-Azinodi-(3-ethyl-benzthiazolinsulfonat-(6)) (ABTS) als Wasserstoff-Donator bei verschiedenen $\mathrm{pH}$-Werten. Zudem werden die Reaktionsbedingungen für die Aktivitätsbestimmung der Peroxidase optimiert.

ABTS ist eines der von S.Hünig (1-3) synthetisierten und polarographisch untersuchten heterocyclischen Azine, 
die als Redoxsysteme drei Oxidationsstufen aufweisen: In einem ersten Schritt wird das meist farblose Azin zu einem stabilen, stark gefärbten Radikalkation oxidiert und bei einem weiteren Elektronenentzug zum Azodikation überführt. Beim ABTS erfolgt der zweite Oxidationsschritt vom grünen Radikalkation zum roten Dikation nur bei großem Uberschuß an Oxidationsmittel und in stark saurem Reaktionsmilieu.

Da ABTS relativ einfach herzustellen ist und wegen der Sulfonierung eine gute Wasserlöslichkeit aufweist, eignet es sich gut als Redoxindikator für die Cerimetrie (4) wie auch als Chromophor für die photometrische $\mathrm{H}_{2} \mathrm{O}_{2}$-Bestimmung bei Anwesenheit von Peroxidase (5-12).

Die Peroxidase ist eines der meist untersuchten Enzyme (Übersichtsartikel: 1. c. (14)), dessen Proteinstruktur in der Zwischenzeit aufgeklärt werden konnte (15). Trotzdem bestehen noch immer offene Fragen bezüglich des Reaktionsmechanismus und der kinetischen Eigenschaften. Für $R$. Childs (16), der den Reaktionsmechanismus der Peroxidase mit dem Redoxindikator ABTS untersuchte, bleibt die Wirkungsweise dieses Enzyms weiterhin komplex und ungeklärt. J. Shindler, der mit $\mathrm{H}_{2} \mathrm{O}_{2}$ und ABTS die Kinetik der Lactoperoxidase (17) sowie die Kinetik der Peroxidase aus menschlichem Cervicalschleim (18) untersuchte, konnte weder für $\mathrm{H}_{2} \mathrm{O}_{2}$ noch für ABTS die Michaelis-Konstanten bestimmen.

\section{Material und Methoden}

Die Peroxidase aus Meerrettich (Donor: hydrogen peroxide oxidoreductase, EC 1.11.1.7) wurde in lyophilisierter Form von Boehringer, Mannheim bezogen. Die Reinheitszahl wurde mit "ca. 3,0" angegeben.

ABTS (kristallisiertes Diammoniumsalz der 2,2'-Azino-di-(3-ethylbenzthiazolinsulfonsäure-(6)) wurde von Sigma und von Boehringer, Mannheim bezogen. Beide Produkte wurden ohne weitere Reinigung eingesetzt, obwohl ca. 1-3\% der Substanz zum grünen ABTS-Radikalkation oxidiert war.

$\mathrm{H}_{2} \mathrm{O}_{2}$ wurde in einer $300 \mathrm{~g} / \mathrm{kg}$ Lösung von der Firma Lehner AG, Muttenz, Schweiz geliefert.

Die übrigen Chemikalien waren von analy tischer Reinheit.

Zur Bestimmung der Peroxidaseaktivität werden in einer Meßzelle von $10 \mathrm{~mm}$ Schichtdicke zu 2,0 ml Peroxidase-Testlösung $(0,1 \mathrm{~mol} / 1$ Natriumacetat, $0,05 \mathrm{~mol} / 1$ Natriumdihydrogenphosphat, $2,0 \mathrm{mmol} / \mathrm{l} \mathrm{ABTS}$ und $2,5 \mathrm{mmol} / 1 \mathrm{H}_{2} \mathrm{O}_{2}$ vom $\mathrm{pH} \mathrm{4,2)}$ $0,05 \mathrm{ml}$ Peroxidaselösung (Peroxidase-Konzentration in der Testlösung: 10 bis $500 \mathrm{ng} / \mathrm{l})$ zugemischt und bei $25^{\circ} \mathrm{C}$ die $\mathrm{Ab}$ sorptionszunahme während 10 bis 60 Minuten photometrisch bei der Wellenlänge $414 \mathrm{~nm}$ gemessen. Abweichungen von diesem Testvorgehen - besonders bezüglich der Zusammensetzung der Testlösung - werden bei den einzelnen Versuchen speziell erwähnt.

\section{Resultate und Diskussion}

Spektren des reduzierten ABTS sowie des ABTS-Radikalkations

$1 \mathrm{~mol} \mathrm{H} \mathrm{O}_{2}$ oxidiert unter der katalytischen Einwirkung von Peroxidase $2 \mathrm{~mol}$ reduziertes ABTS (ABTS) zum stabilen, grünen ABTS-Radikalkation $\left(\mathrm{ABTS}^{+}\right)$.

$$
\mathrm{H}_{2} \mathrm{O}_{2}+2 \mathrm{ABTS}+2 \mathrm{H}^{+} \stackrel{\text { Peroxidase }}{\longrightarrow} 2 \mathrm{H}_{2} \mathrm{O}+2 \mathrm{ABTS}^{+}
$$

Das Spektrum dieses ABTS-Radikalkations ist in Abbildung 1 aufgezeichnet. Es zeigt, daß die grüne Farbe aus einer Mischung von Gelb und Blau entsteht. Das Absorptionsmaximum liegt bei 414nm. Demgegenüber ist die Absorption bei $405 \mathrm{~nm}$ um 7\%, bei $578 \mathrm{~nm}$ um $75 \%$ und bei 650 nm um 63\% geringer, was sich entsprechend auf die Sensitivität der Aktivitätsbestimmung der Peroxidase auswirkt. Für die weiteren Versuche wurde die Farbintensität des ABTS-Radikalkations bei der Wellenlänge $414 \mathrm{~nm}$ gemessen.

Das reduzierte ABTS ist farblos, sein Absorptionsmaximum liegt bei der Welleniänge $340 \mathrm{~nm}$.

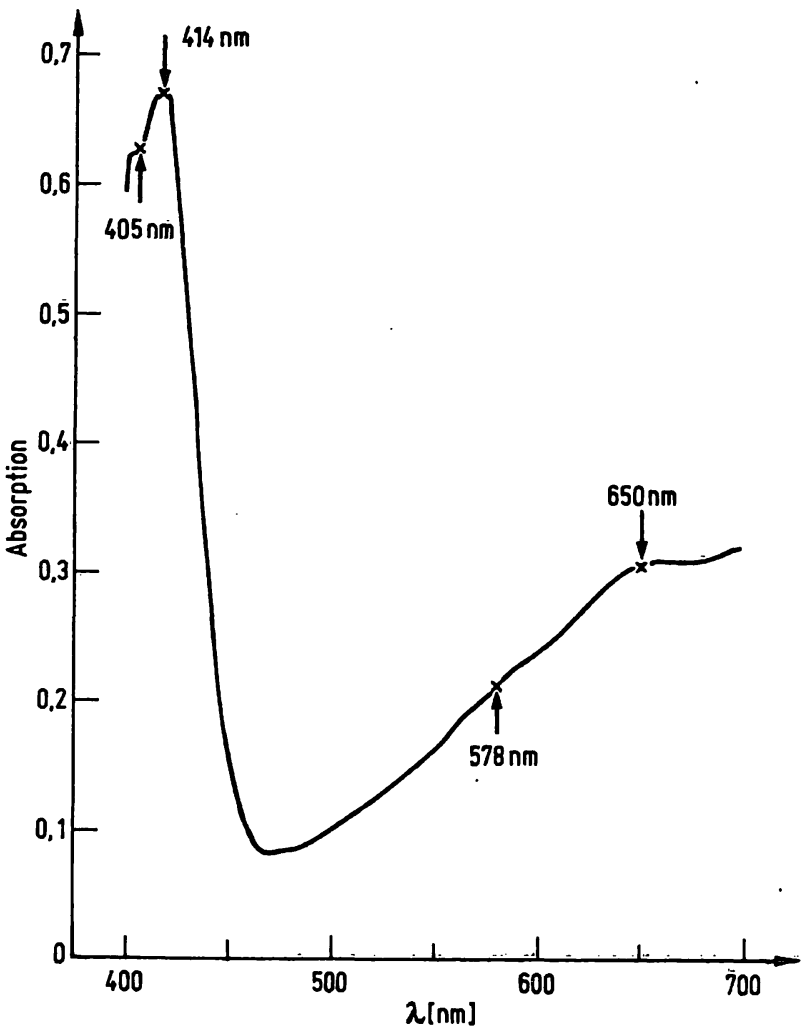

Abb. 1. Absorptionsspektrum des ABTS-Radikalkations (ABTS ${ }^{+}$). Nach der katalytischen Einwirkung von $200 \mathrm{ng} / 1$ Peroxidase auf $2 \mathrm{mmol} / 1 \mathrm{ABTS}$ und $2.5 \mathrm{mmol} / 1 \mathrm{H}_{2} \mathrm{O}_{2}$ in $0,1 \mathrm{~mol} / 1$ Natriumacetat mit $0,05 \mathrm{~mol} / 1 \mathrm{Natriumdihydro}$ genphosphat während 60 Minuten bei $25^{\circ} \mathrm{C}$ wird das Spektrum des gebildeten ABTS-Radikalkations gegen den Reagenzienleerwert im Wellenlängenbereich von 400 $700 \mathrm{~nm}$ aufgenommen.

\section{Peroxidaseaktivität in Abhängigkeit zur ABTS-Konzen- tration bei verschiedenen $\mathrm{pH}$-Werten}

Um einen genaueren Einblick in die Besonderheiten der Peroxidase aus Meerrettich zu erhalten, wurde zunächst bei gegebener $\mathrm{H}_{2} \mathrm{O}_{2}$-Konzentration von $2,5 \mathrm{mmol} / 1$ die Wirkung der ABTS-Konzentration bei unterschiedlichen pH-Werten auf die Peroxidaseaktivität untersucht. Die dabei erhalțenen Resultate sind in den Abbildungen 2 und 3 zusammengefaßt. Daraus ist ersichtlich, daß sowohl die maximal mögliche Reaktionsgeschwindigkeit der Peroxidase wie auch die dafür notwendige optimale ABTS- 


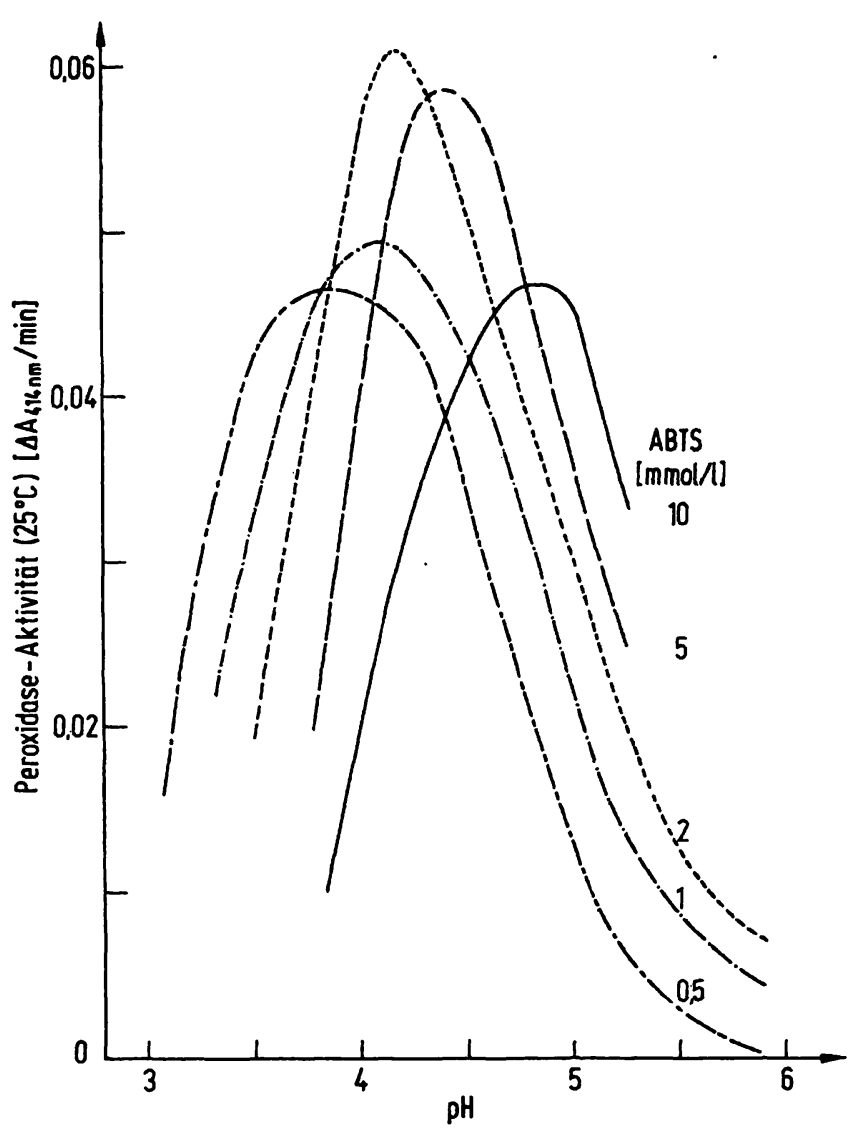

Abb. 2. pH-Aktivitätskurve der Peroxidase in Abhängigkeit zur ABTS-Konzentration.

Aktivitätsbestimmung von $0,5 \mu \mathrm{g} / 1$ Peroxidase in $0,1 \mathrm{~mol} / 1 \mathrm{Natriumacetat,} 0,05 \mathrm{~mol} / 1$ Natriumdihydrogenphosphat, $2,5 \mathrm{mmol} / 1 \mathrm{H}_{2} \mathrm{O}_{2}$ und den entsprechenden ABTS-Konzen trationen bei den angegebenen pH-Werten. Reaktionstemperatur: $25^{\circ} \mathrm{C}$.

Konzentration vom $\mathrm{pH}$-Wert abhängig ist. Bei geringeren pH-Werten genügen nach dem Kurvenverlauf der Abbildung 2 geringere ABTS-Konzentrationen zur Sättigung des vorhandenen Enzyms und zur Erreichung der bei diesen pH-Werten maximal möglichen Reaktionsgeschwindigkeit. Mit steigendem pH-Wert ist eine größere ABTS-

Menge zur vollen Enzymsättigung notwendig. Dies besagt, daß die ABTS-Affinität der Peroxidase mit steigendem pH-Wert abnimmt. Die höchste Peroxidaseaktivität wird unter den angegebenen Reaktionsbedingungen beim pH 4,2 mit einer ABTS-Konzentration von 2 mmol/1 erreicht. Die einzelnen pH-Optima der Enzymaktivität werden mit zunehmender ABTS-Konzentration gegen den Neutralpunkt verschoben.

In Abbildung 3 ist die Abhängigkeit der Peroxidaseaktivität von der $\mathrm{ABTS}$-Konzentration bei den verschiedenen pH-Werten nach der Darstellungsweise von Lineweaver: Burk zusammengefaßt. Daraus kann direkt die ABTSAffinität der Peroxidașe als $K_{\mathrm{m}}$ wie auch die maximal mögliche Reaktionsgeschwindigkeit bei den betreffenden pH-Werten abgelesen werden.

Die Abbildung 4 żeigt die Korrelation der ABTS-Affinität $\left(\mathrm{p} K_{\mathrm{m}}=-\log K_{\mathrm{m}}\right)$ der Peroxidase $z u$ den entsprechen-

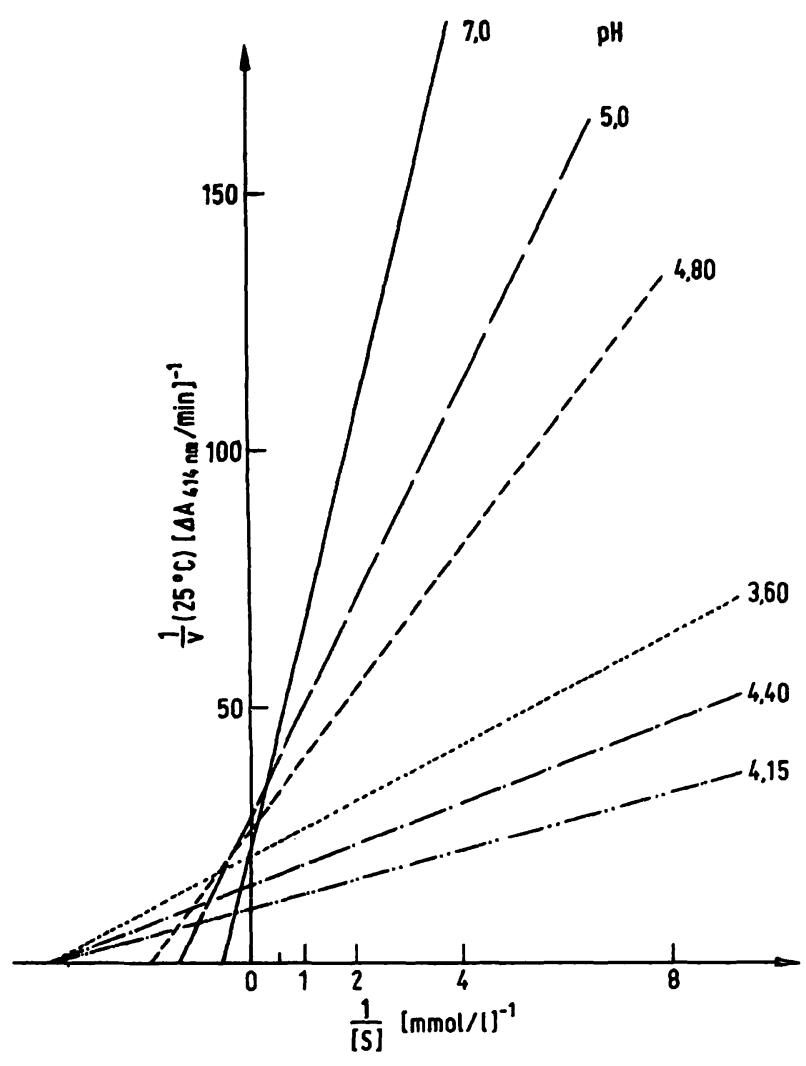

Abb. 3. Michaelis-Konstante der Peroxidase für ABTS bei verschiedenen $\mathrm{pH}$-Werten.

Aktivitätsbestimmung von $0,5 \mu \mathrm{g} / 1$ Peroxidase in $0,1 \mathrm{~mol} / 1$ Natriumacetat, $0,05 \mathrm{~mol} / 1$ Natriumdihydrogenphosphat, $2,5 \mathrm{mmol} / 1 \mathrm{H}_{2} \mathrm{O}_{2}$ und den entsprechenden ABTS-Kon= zentrationen bei den angegebenen pH-Werten. Reaktionstemperatur: $25^{\circ} \mathrm{C}$. Darstellung nach Lineweaver-Burk.

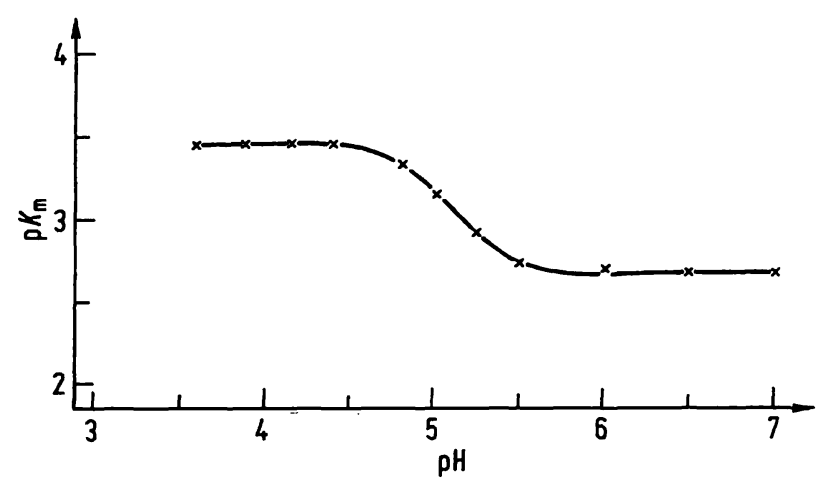

Abb. 4. Abhängigkeit der $\mathrm{p} K_{\mathrm{m}}$-Werte vom $\mathrm{pH}$ für $\mathrm{ABTS}$. Die aus der Abbildung 3 errechneten Michaelis Konstanten $K_{\mathrm{m}}$ werden als $\mathrm{p} K_{\mathrm{m}}\left(=-\log K_{\mathrm{m}}\right)$ in Abhängigkeit zum pH dargestellt.

den $\mathrm{pH}-$ Werten. Dieser Kurvenverlauf bedeutet nach M. Dixon (19), daß durch die ABTS-Peroxidase-Komplexbildung die Ionisierung der für die Enzymaktivität entscheidenden Gruppe der Peroxidase zunimmt.

Peroxidaseaktivität in Abhängigkeit zur $\mathrm{H}_{2} \mathrm{O}_{2}$-Konzentration bei verschiedenen $\mathrm{pH}$-Werten

Bei Anweșenheit von $2 \mathrm{mmol} / \mathrm{l}$ ABTS wurde die Abhängigkeit der Peroxidaseaktivität von der $\mathrm{H}_{2} \mathrm{O}_{2}$-Konzen- 


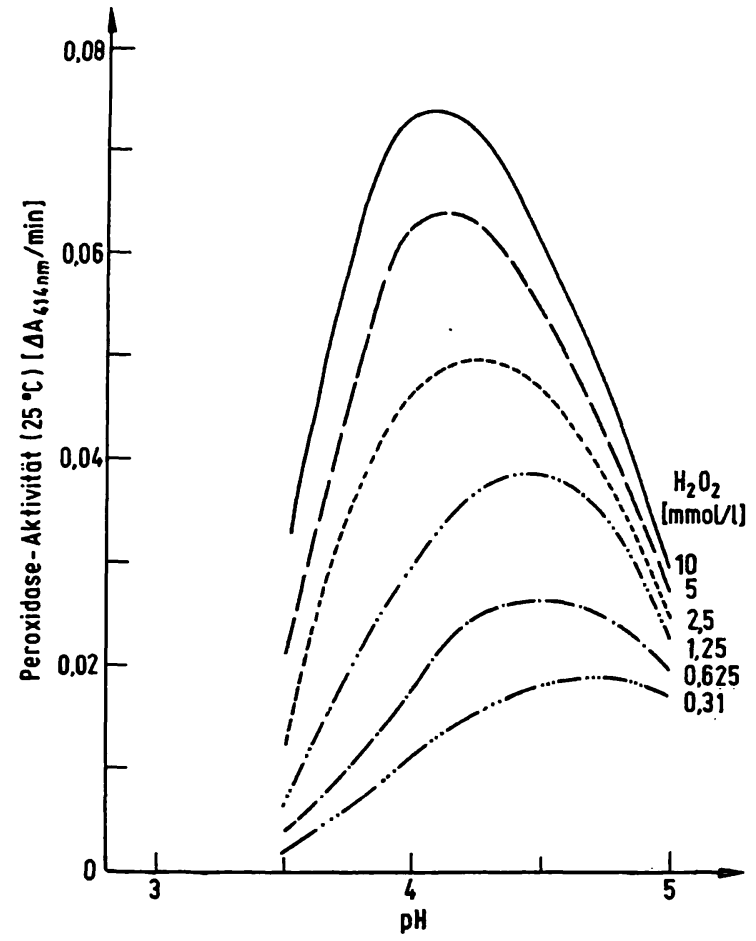

Abb. 5. pH-Aktivitätskurve der Peroxidase in Abhängigkeit zur $\mathrm{H}_{2} \mathrm{O}_{2}$-Konzentration.

Aktivitätsbestimmung von $0,5 \mu \mathrm{g} / 1$ Peroxidase in $0,1 \mathrm{~mol} / \mathrm{t}$ Natriumacetat, 0,05 mol/1 Natriumdihydrogenphosphat, $2 \mathrm{mmol} / \mathrm{l} \mathrm{ABTS}$ und den entsprechenden $\mathrm{H}_{2} \mathrm{O}_{2}$-Konzentrationen bei den angegebenen pH-Werten. Reaktionstemperatur: $25^{\circ} \mathrm{C}$.

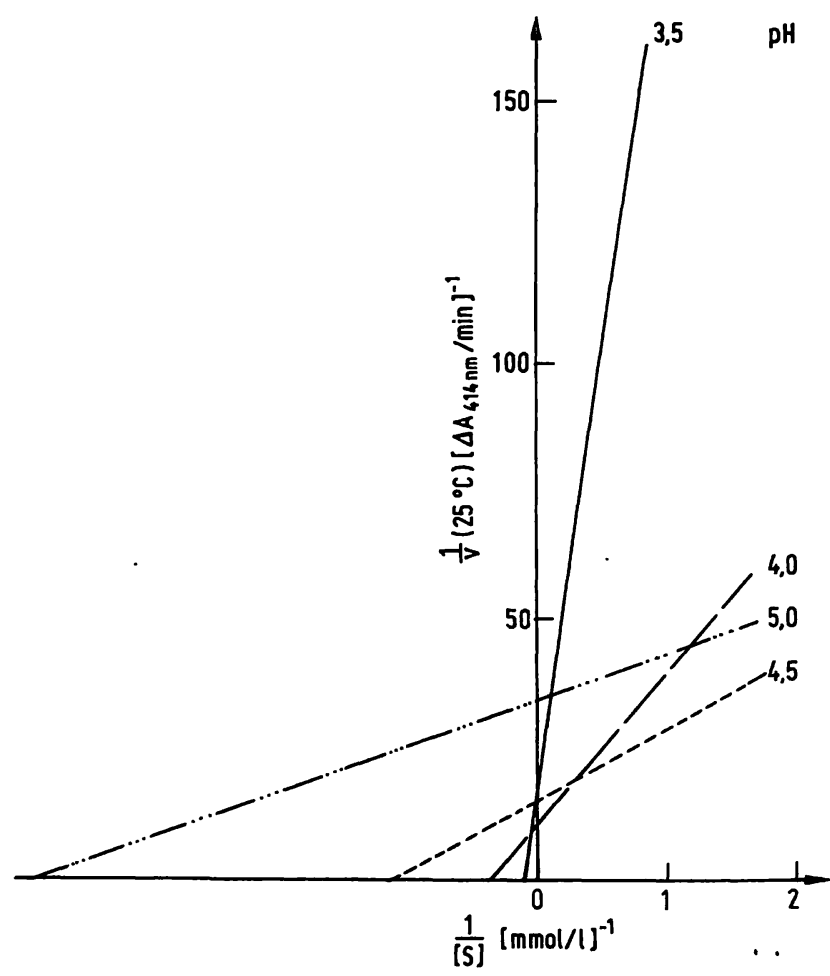

Abb. 6. Michaelis Konstante der Peroxidase für $\mathrm{H}_{2} \mathrm{O}_{2}$ bei verschiedenen pH-Werten.

Aktivitätsbestimmung von $0,5 \mu \mathrm{g} / 1$ Peroxidase in $0,1 \mathrm{~mol} / 1$ Natriumace tat, $0,05 \mathrm{~mol} / 1$ Natriumdihydrogenphosphat, $2 \mathrm{mmol} / \mathrm{l}$ ABTS und den entsprechenden $\mathrm{H}_{2} \mathrm{O}_{2}$-Konzentrationen bei den angegebenen $\mathrm{pH}$-Werten. Reaktionstemperatur: $25^{\circ} \mathrm{C}$. Darstellung nach Lineweaver-Burk. tration und dem pH-Wert untersucht. Die entsprechenden Resultate sind in den Abbildungen 5 und 6 zusammengefaßt.

Die Abbildung 5 żeigt eine deutliche Verschiebung der pH-Optima der Peroxidaseaktivität bei abnehmender $\mathrm{H}_{2} \mathrm{O}_{2}$-Konzentration gegen den Neutralpunkt hin. Im Gegensatz zum ABTS nimmt die $\mathrm{H}_{2} \mathrm{O}_{2}$-Affinität der Peroxidase mit steigendem $\mathrm{pH}$-Wert wesentlich $\mathrm{zu}$, was vor allem deutlich wird, wenn diese Resultate nach der Darstellungsweise von Lineweaver-Burk wiedergegeben werden (Abb. 6). Im untersuchten pH-Bereich nimmt die $\mathrm{H}_{2} \mathrm{O}_{2}$-Affinität zur Peroxidase pro $\mathrm{pH}$-Einheit um das zehnfache $\mathrm{zu}$, wie dies aus der $\mathrm{p} K_{\mathrm{m}}: \mathrm{pH}-$ Korrelation ersichtlich wird (Abb. 7). Dieser $\mathrm{pK}_{\mathrm{m}}-\mathrm{pH}$-Kurvenverlauf kann auf folgende Weise interpretiert werden: In ähnlicher Weise wie Wasser dissoziert auch das $\mathrm{H}_{2} \mathrm{O}_{2} \cdot \mathrm{zu} \mathrm{H}^{+}$ und $\mathrm{O}_{2} \mathrm{H}^{\prime}$. Bei einer vorgegebenen $\mathrm{H}_{2} \mathrm{O}_{2}$-Konzentration nimmt dementsprechend mit steigendem $\mathrm{pH}$-Wert die $\mathrm{O}_{2} \mathrm{H}^{-}-$Konzentration gleichsinnig zu. Da nun aber anzunehmen ist, daß das $\mathrm{O}_{2} \mathrm{H}^{-}$als das eigentliche Substrat der Peroxidase zu betrachten ist, muß zur Aufrechterhaltung einer gleichbleibenden $\mathrm{O}_{2} \mathrm{H}^{-}-$Konzentration in der Testlösung bei tieferen pH-Werten eine entsprechend höhere $\mathrm{H}_{2} \mathrm{O}_{2}$ - Konzentration eingesetzt werden. Die Erhöhung der $\mathrm{H}_{2} \mathrm{O}_{2}$-Affinität der Peroxidase mit steigendem $\mathrm{pH}$-Wert ist demnach nur eine scheinbare, die eigentliche Substrataffinität $\left(\mathrm{O}_{2} \mathrm{H}\right)$ der Peroxidase wird innerhalb des untersuchten $\mathrm{pH}$-Bereichs vom $\mathrm{pH}$ nicht beeinflußt.

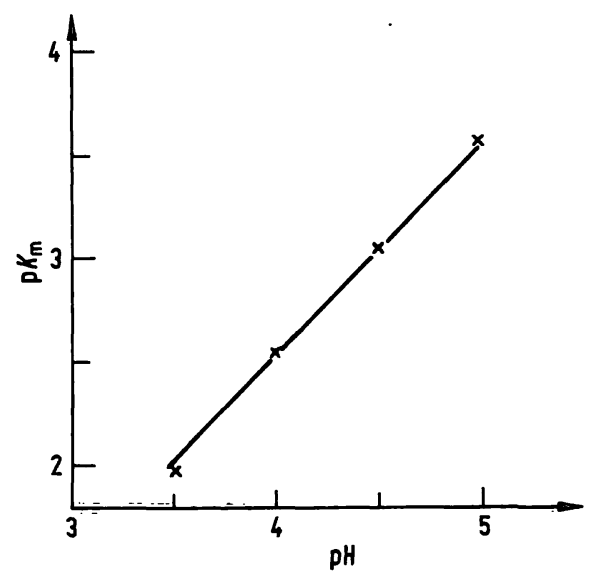

Abb. 7. Abhängigkeit der $\mathrm{p} K_{\mathrm{m}}$-Werte für $\mathrm{H}_{2} \mathrm{O}_{2}$ vom $\mathrm{pH}$. Die aus der Abbildung $\sigma$ errechneten Michaeliskonstanten $K_{\mathrm{m}}$ werden als $\mathrm{p} K_{\mathrm{m}}\left(=-\log K_{\mathrm{m}}\right)$ in Abhängigkeit zum $\mathrm{pH}$ aufgetragen.

Gegenseitige Beeinflussung von $\mathrm{ABTS}$ und $\mathrm{H}_{2} \mathrm{O}_{2}$ bezüglich der Peroxidase-Affinität

Für die katalytische Reaktion müssen sowohl ABTS wie auch $\mathrm{H}_{2} \mathrm{O}_{2}$ von der Peroxidase komplex gebunden werden. Um die gegenseitige Beeinflussung dieser beiden Substrate bei der Komplexbildung mit dem Enżyym żu untersuchen, wurde die Peroxidaseaktivität bei sonst. 
identischen Reaktionsbedingungen mit unterschiedlichen ABTS- und $\mathrm{H}_{2} \mathrm{O}_{2}$ - Konzentrationen bestimmt. Die dabei erhaltenen Resultate, die in der Tabelle $1 \mathrm{zu}$ sammengefaßt sind, zeigen deutlich, daß ABTS und $\mathrm{H}_{2} \mathrm{O}_{2}$ bei der Komplexbildung mit der vorhandenen Peroxidase stark konkurrieren. Die Erhöhung der Konzentration des einen Substrates (ABTS oder $\mathrm{H}_{2} \mathrm{O}_{2}$ ) senkt die Affinität des anderen Substrates und erschwert dementsprechend die Komplexbildung mit der Peroxidase. Die MichaelisKonstante $K_{\mathrm{m}}$ des einen Substrates wird größer, je mehr vom anderen Substrat in der Testlösung enthalten ist. Dabei ist allerdings zu betonen, daß die kataly tische Reaktionsgeschwindigkeit nicht direkt abhängig ist von der Substrataffinität. Die größtmögliche Peroxidaseaktivität wird bei einem optimalen Konzentrationsverhältnis der beiden Substrate ABTS und $\mathrm{H}_{2} \mathrm{O}_{2}$ erreicht.

Tab. 1. Michaelis-Konstanten für ABTS und $\mathrm{H}_{2} \mathrm{O}_{2}$ in gegenseitiger Abhängigkeit

In $0,1 \mathrm{~mol} / 1 \mathrm{Natriumacetat}$ und $0,05 \mathrm{~mol} / 1 \mathrm{Natriumdihy}-$ drogenphosphat vom pH 4,2 wird die Aktivität von $0,5 \mu \mathrm{g} / 1$ Peroxidase in Anwesenheit unterschiedlicher ABTS- und $\mathrm{H}_{2} \mathrm{O}_{2}$-Konzentrationen bei einer Reaktionstemperatur von $25^{\circ} \mathrm{C}$ photometrisch gemessen und die entsprechenden Michaelis-Konstanten für ABTS und $\mathrm{H}_{2} \mathrm{O}_{2}$ bestimmt.

\begin{tabular}{ll||ll}
\hline $\begin{array}{l}\mathrm{H}_{2} \mathrm{O}_{2} \\
(\mathrm{mmol} / \mathrm{l})\end{array}$ & $\begin{array}{l}K_{\mathrm{m}} \text { für ABTS } \\
(\mathrm{mmol} / \mathrm{l})\end{array}$ & $\begin{array}{l}K_{\mathrm{m}} \text { für } \mathrm{H}_{2} \mathrm{O}_{2} \\
(\mathrm{mmol} / \mathrm{l})\end{array}$ & $\begin{array}{l}\mathrm{ABTS} \\
(\mathrm{mmol} / \mathrm{l})\end{array}$ \\
\hline 10,0 & 0,66 & 1,56 & 2,0 \\
5,0 & 0,50 & 1,0 & 1,0 \\
2.5 & 0,24 & 0,30 & 0,5 \\
1,25 & 0,15 & 0,158 & 0,25 \\
\hline
\end{tabular}

\section{Einfluß des Puffersystems auf die Aktivität der Peroxi- dase}

Als günstigstes Puffersystem erweist sich $0,1 \mathrm{~mol} / 1$ Natriumacetat, dem zur Stabilisierung des Reagenzienleerwertes $0,05 \mathrm{~mol} / 1$ Natriumdihydrogenphosphat zugesetzt werden. Demgegenüber ist unter sonst identischèn Reaktionsbedingungen ( $2 \mathrm{mmol} / \mathrm{ABTS}, 2,5 \mathrm{mmol} / 1 \mathrm{H}_{2} \mathrm{O}_{2}$, $\mathrm{pH} 4,2$ und $25^{\circ} \mathrm{C}$ ) die Peroxidaseaktivität zu Beginn der katalytischen Reaktion in $0,1 \mathrm{~mol} / 1$ Natriumcitrat um $11 \%$, in $0,1 \mathrm{~mol} / 1 \mathrm{~L}$-Tartrat um $25 \%$ und in $0,1 \mathrm{~mol} / 1$ Natriumphthalat um $44 \%$ geringer. Zudem nimmt in dieser gleichen Reihenfolge die Peroxidase-Inaktivierung während der Reaktionsdauer zụ, so daß die Aktivitätsunterșchiede immer größer werden, je länger die Peroxidase in den entsprechenden Testlösungen inkubiert wird. Die Konzentration an Natriumacetat hat im Bereich von 50 bis $200 \mathrm{mmol} / 1$ bei Anwesenheit von $50 \mathrm{mmol} / 1$ Natriumdihydrogenphosphat keinen Einfluß weder auf die Aktivität noch auf die Inaktivierungsgeschwindigkeit der Peroxidase.

Ammoniumionen, welche die Aktívität der Meerrettich Peroxidase um das 160-fache ạktivieren, wenn sie bei hö- heren $\mathrm{pH}$-Werten mit den Substraten $\mathrm{H}_{2} \mathrm{O}_{2}$ und $o$-Dianisidin bestimmt wird (20), haben bei pH 4,2 bei Anwesenheit der Substrate $\mathrm{H}_{2} \mathrm{O}_{2}$ und ABTS keinen Einfluß auf die Reaktionsgeschwindigkeit der Peroxidase.

\section{Einfluß der Reaktionsdauer auf die Aktivität der Peroxi- dase}

Da bei den enzym-immunologischen Tests kleinste Mengen Peroxidase nachgewiesen und daher das Enzym längere Zeit in der Testlösung inkubiert werden muß, ist es notwendig, die Peroxidaseaktivität auch in Abhängigkeit zur Reaktionsdauer zu untersuchen. Aus den diesbezüglichen Resultaten, die in der Abbildung 8 zusammengestellt sind, wird deutlich, daß fur die Optimierung der Reaktionsbedingungen bei der Peroxidase auch der Zeitfaktor miteinbezogen werden muß. Je nach Reaktionsdauer ändert sich die optimale $\mathrm{H}_{2} \mathrm{O}_{2}$-Konzentration. So wird bei Anwesenheit von $2 \mathrm{mmol} / \mathrm{l}$ ABTS die höchste

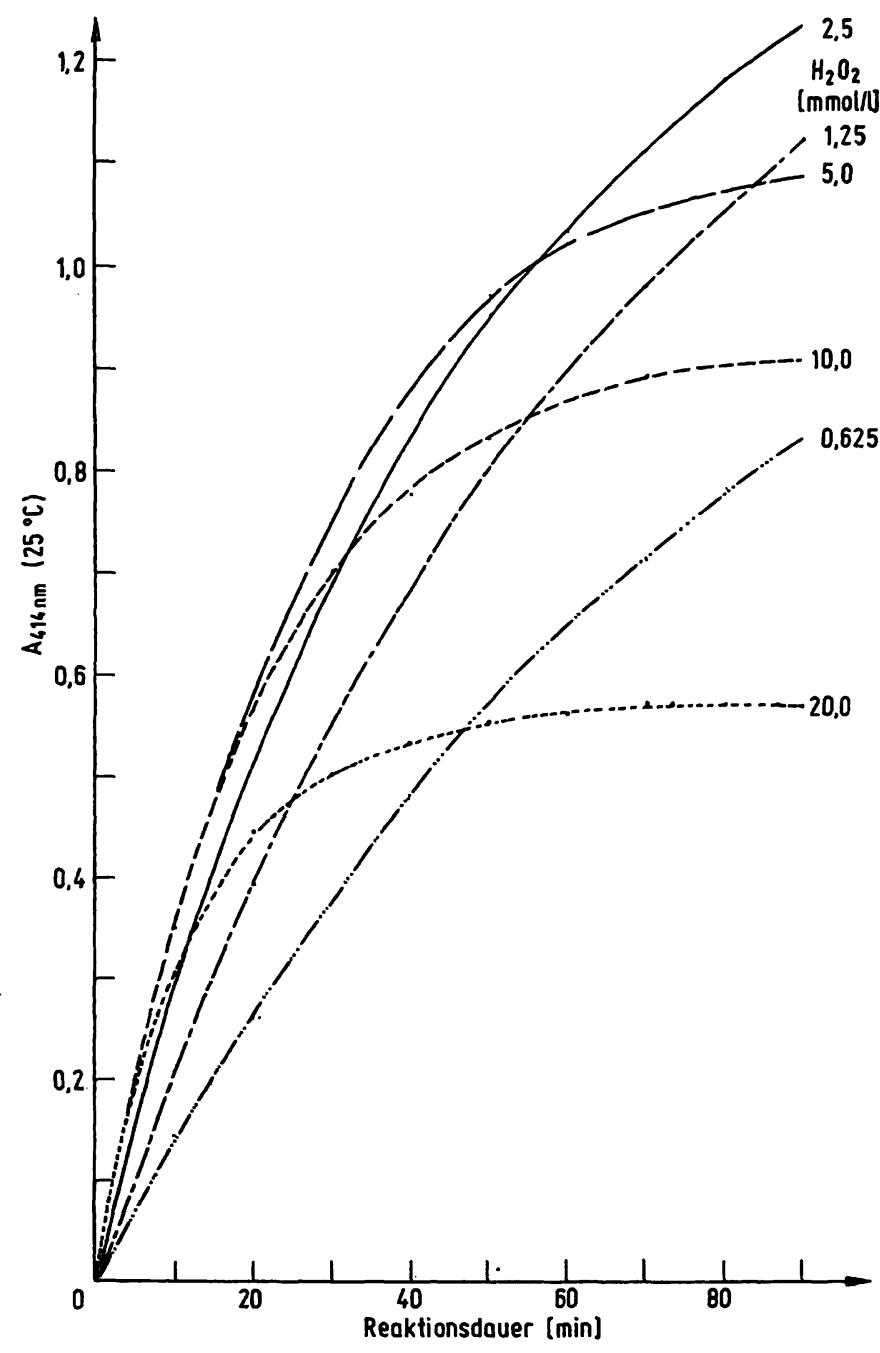

Abb. 8. Peroxidạaseaktivität in Abhängigkeit zur $\mathrm{H}_{2} \mathrm{O}_{2}$-Konzentration und zur Reaktionsdauer.

Bei einer Reaktionstemperatur von $25^{\circ} \mathrm{C}$ werden $250 \mathrm{ng} / 1$ Peroxidase in $0,1 \mathrm{~mol} / \mathrm{l}$ Natriumacetat vom pH 4,2 mit 0,05 mol/1 Natriumdihydrogenphosphat, $2 \mathrm{mmol} / \mathrm{l}$ ABTS und verschiedenen $\mathrm{H}_{2} \mathrm{O}_{2}$-Konzentrationen inkubiert und die Absorption bei der Wellenlänge $414 \mathrm{~nm}$ während 90 Minuten verfolgt. 
Anfangsaktivität der Peroxidase mit $20 \mathrm{mmol} / 1 \mathrm{H}_{2} \mathrm{O}_{2}$ gemessen. Bei einer Reaktionsdauer von 10 Minuten werden $10 \mathrm{mmol} / 1 \mathrm{H}_{2} \mathrm{O}_{2}$, bei einer solchen von 20 Minuten $5 \mathrm{mmol} / 1 \mathrm{H}_{2} \mathrm{O}_{2}$ und bei einer Reaktionsdauer über 60 Minuten $2,5 \mathrm{mmol} / 1 \mathrm{H}_{2} \mathrm{O}_{2}$ eingesetzt, um unter sonst identischen Bedingungen die höchste Farbintensität zu erreichen. Diese Verhältnisse sind damit zu erklären, daß mit zunehmender $\mathrm{H}_{2} \mathrm{O}_{2}$-Konzentration in der Testlösung die Peroxidase entsprechend schneller inaktiviert wird. Diese Inaktivierung wird nur durch das $\mathrm{H}_{2} \mathrm{O}_{2}$ verursacht und kann durch die Anwesenheit auch größerer Konzentrationen von $A B T S$ nicht verhindert noch verringert werden. ABTS bietet der Peroxidase keinen Schutz vor der $\mathrm{H}_{2} \mathrm{O}_{2}$-Inaktivierung, obwohl es zusammen mit dem $\mathrm{H}_{2} \mathrm{O}_{2}$ komplex ans aktive Zentrum des Enzyms gebunden wird.

\section{Einfluß von Serum-Protein auf die Peroxidaseaktivität}

$\mathrm{Da}$ bei der vorgeschlagenen Peroxidase-Testmethode bei einem $\mathrm{pH}$-Wert von 4,2 gearbeitet wird, ist zu erwarten, daß die Serum-Proteine in einer größeren Konzentration ausfallen. Diese Testmethode ist nicht vorgesehen und auch nicht geeignet, die Peroxidaseaktivität im Serum zu bestimmen. Dazu müßte die Testlösung dahingehend abgeändert werden, daß die Serumprobe in $0,1 \mathrm{~mol} / 1 \mathrm{Natri-}$ umacetat vom $\mathrm{pH} 5,0 \mathrm{mit} 0,05 \mathrm{~mol} / 1$ Natriumdihydrogenphosphat, $10 \mathrm{mmol} / \mathrm{l} \mathrm{ABTS}$ und $1,25 \mathrm{mmol} / 1 \mathrm{H}_{2} \mathrm{O}_{2}$ bei $25^{\circ} \mathrm{C}$ inkubiert wird.

Serum-Protein, das bis zu einer Konzentration von $100 \mathrm{mg} / \mathrm{l}$ in der Peroxidase-Testlösung vom $\mathrm{pH} 4,2$ löslich ist, vermindert die meßbare Peroxidase-Aktivität (Tab. 2). Dabei tritt das Protein als Wasserstoffdonator in Konkurrenz zum ABTS, wodurch weniger ABTS-Radikalkation gebildet wird. Dies führt zu einer entsprechend geringeren Farbentwicklung.

Tab. 2. Einfluß von Serumprotein auf die Aktivität der Peroxidase Bei Anwesenheit verschiedener Serumproteinmengen in der Testlösung wurde die katalytische Wirkung von $500 \mathrm{ng} / \mathrm{l}$ Peroxidase in $0,1 \mathrm{~mol} / 1$ Natriumacetat vom $\mathrm{pH} \mathrm{4,2} \mathrm{mit}$ $0,05 \mathrm{~mol} / 1$ Natriumdihydrogenphosphat, $2 \mathrm{mmol} / 1 \mathrm{ABTS}$ und $2,5 \mathrm{mmol} / 1 \mathrm{H}_{2} \mathrm{O}_{2}$ bestimmt, indem bei der Reaktionstemperatur von $25^{\circ} \mathrm{C}$ die Absorptionsdifferenz während den ersten 10 Minuten bei der Wellenlänge $414 \mathrm{~nm}$ gemessen wurde. Durch Zusatz einer entsprechenden Menge Humanserum wurde die gewünschte Serumproteinkonzentration erreicht.

\begin{tabular}{cll}
\hline $\begin{array}{l}\text { Serumprotein } \\
\text { in der Testlösung } \\
(\mathrm{mg} / \mathrm{l})\end{array}$ & $\begin{array}{l}\text { Peroxidase-Aktivität } \\
\left(\Delta \mathbf{A}_{414 \mathrm{~nm}} / \mathrm{min}\right) \\
\left(25^{\circ} \mathrm{C}\right)\end{array}$ & $\begin{array}{l}\text { Peroxidase- } \\
\text { Aktivität } \\
(\%)\end{array}$ \\
\hline 0 & 0,0580 & 100 \\
12,5 & 0,0560 & 97 \\
25,0 & 0,0530 & 91 \\
50,0 & 0,0520 & 90 \\
100,0 & 0,0500 & 86 \\
\hline
\end{tabular}

Da bei den enzym-immunologischen Bestimmungen sowohl die Peroxidase wie auch die übrige Proteinkonzentration (Antigen oder Antikörper) bei der enzymatischen Indikatorreaktion äußerst klein ist, kann die aufgezeigte Proteininterferenz bei diesen Peroxidașe-Aktivitätsbestimmungen vernachlässigt werden.

\section{Empfindlichkeit der beschriebenen Methode zur Be- stimmung der Peroxidaseaktivität}

Die Abbildung 9 zeigt die lineare Proportionalität der Peroxidaseköonzentration žur gemessenen Absorptionsdifferenz pro Zeiteinheit. Die Nachweisgrenze liegt bèi $10 \mathrm{ng} / \mathrm{l}$ Peroxidase. Anderseits könnte der Konzentrationsbereich wesentlich über die $250 \mathrm{ng} / \mathrm{l}$ erweitert werden bei entsprechend verkürzten Reaktionszeiten. $\mathrm{Da}$ bei den enzym-immunologischen Bestimmungen mit Standards gearbeitet wird, kommt der Berechnung der Peroxidasekonzentration aus der gemessenen Absorptionsdifferenz pro Zeiteinheit nur untergeordnete Bedeutung zu. Diese könnte mit Hilfe einer Peroxidase-Standardkurve (Abb. 9) oder bei einer genau definierten Bestimmungsmethode faktoriell über den molaren Absorptionskoeffizienten des ABTS-Radikalkations erfolgen.

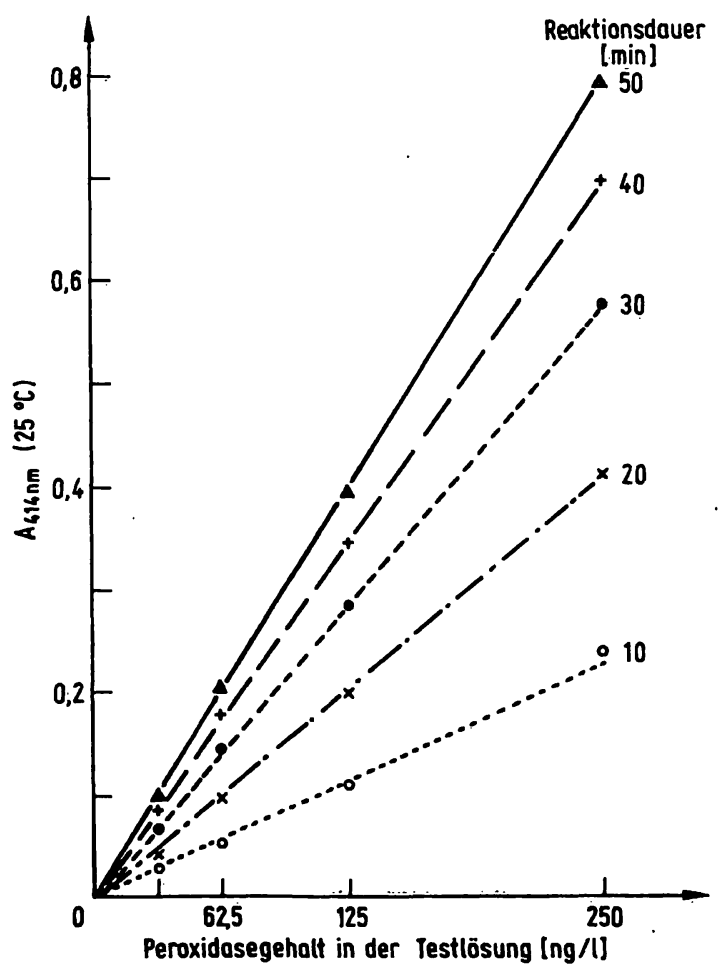

Abb. 9. Abhängigkeit der Absorptionsdifferenz von der Peroxidasekonzentration und der Reaktionsdauer. Die angegebenen Peroxidasemengen werden in $0,1 \mathrm{~mol} / \mathrm{l}$ Natriumáce tat vom pH 4,2 mit 0,05 mol/1 Natriumdihy̆drogenphosphat, $2 \mathrm{mmol} / \mathrm{l} \mathrm{ABTS}$ und $2 ; 5 \mathrm{mmol} / \mathrm{l}$ $\mathrm{H}_{2} \mathrm{O}_{2}$ während 50 Minuten bei einer Reaktionstemperatur von $25^{\circ} \mathrm{C}$ inkubiert und dabei laufend die Absorptionszunahme bei dẹ Wellenlänge $414 \mathrm{~nm}$ gemessen. 


\section{Literatur}

1. Hünig, S. (1964), Ann. Chem. 676, 32-35.

2. Hünig, S., Balli, H., Conrad, H. \& Schott, A. (1964), Ann. Chem. 676, 36-51.

3. Hünig, S., Balli, H., Conrad, H. \& Schott, A. (1964), Ann. Chem. 676, 52-65.

4. Lang, H., Hönel, H. \& Hahn, H. (1964), Z. Anal. Chem. 201, $321-325$.

5. Gawehn, K., Wielinger H. \& Werner, W. (1970), Z. Anal. Chèm. $252,222-224$.

6. Werner, W., Rey, H.-G. \& Wielinger, H. (1970), Z. Anal. Chem. $252,224-228$.

7. Kahle, K., Weiss, L., Klarwein, M. \& Wieland, O. (1970), Z. Anal. Chem. 252, 228-231.

8. Eberhardt, D., Decker, S. \& Klingmüller, V. (1971), Z. Klin. Chem. Klin. Biochem. 9, 362-363.

9. Susić, D. \& Scheibe, P. (1971), Z. Anal. Chem. 257, 130-132.

10. Schreiber, J. \& Lachenicht, R. (1973), Z. Klin. Chem. Klin. Biochem. 11, 31-34.
11. Szasz, G., Huth, K. \& Busch, E. (1974), Z. Klin. Chem. Klin. Biochem. 12, 256.

12. Uete, T., Hori, T. \& Fukutani, C. (1976), Clin. Chim Acta 70, 329-332.

13. Majkić, N. \& Berkes, I. (1977), Clin. Chim. Acta 80, 121-131.

14. Yamazaki, I. \& Yokota, K. (1973), Molecular Cellular Biochem. 2, 39-52.

15. Welinder, K. G. (1976), FEBS Letters 72, 19-23.

16. Childs, R. E. \& Bardsley, W. G. (1975), Biochem. J. 145 , 93-103.

17. Shindler, J. S. \& Bardsley, W. G. (1975), Biochem. Biophys. Res. Commun. 67, 1307-1312.

18. Shindler, J. S., Childs, R. E. \& Bardsley, W. G. (1976), Eur. J. Biochem. 65, 325-331.

19. Dixon, M. (1953), Biochem. J. 55, 161-170.

20. Fridovich, I. (1963), J. Biol. Chem. 238, 3921-3927.

Dr. H. Gallati

Diag. Forschungsabteilung F. Hoffmann-La Roche \& Co. Grenzacherstr. 124 CH-4002 Basel 
. 